\title{
THE COMPONENTS OF A JULIA SET
}

\author{
A.F. Beardon
}

Let $R$ be a rational map of degree $d$ of the complex sphere $\mathbf{C}_{\infty}$ onto itself, and let $J$ and $F$ be the Julia and Fatou sets of $R$ respectively. We assume throughout that $d \geq 2$; then $J$ is the smallest compact set $E$ which contains at least three points, and which satisfies

$$
R(E)=E=R^{-1}(E) .
$$

We call this property the complete invariance of $E$, and the fact that $J$ is the smallest such set is referred to as the minimality of $J$. For details of the general theory, we refer the reader to [1], [2] and [3]. It is known that $J$ is a perfect set (so $J$ is uncountable, and no point of $J$ is isolated), and also that if $J$ is disconnected, then it has infinitely many components. The following result, which seems not to have been noticed before, contains both of these results (when $J$ is disconnected) and more.

Theorem. If $J$ is disconnected, then it has uncountably many many components, and each point of $J$ is an accumulation point of distinct components of $J$.

In [4], McMullen gives an example in which $J$ has a buried component (that is, a component of $J$ which is not on the boundary of any component of $F$ ). If each component of $F$ has finite connectivity, and if $J$ is disconnected, then there are only countably many components of $J$ which lie on the boundary of some component of $F$, and our Theorem immediately yields the following general result.

Corollary. Suppose that $J$ is disconnected, and that every component of $F$ has finite connectivity. Then $J$ has a buried component.

The major part of the proof of the Theorem is contained in the following

Proposition. Let $K$ be a compact connected subset of $\mathbf{C}_{\infty}$. Then $R^{-1}(K)$ has at most $d$ components, and each is mapped by $R$ onto $K$.

The proof of the Proposition is easier if we first discuss some preliminary results. The complement of a set $A$ with respect to the plane $\mathbf{C}$ and the sphere $\mathbf{C}_{\infty}$ are denoted by $\mathbf{C}-A$ and $\mathbf{C}_{\infty}-A$ respectively. First, we quote 
Lemma 1 ([5], p. 144). A compact subset $W$ of $\mathbf{C}_{\infty}$ is connected if and only if every component of $\mathbf{C}_{\infty}-W$ is simply connected.

Next, consider a bounded domain $D$ in $\mathbf{C}$ which is bounded by a finite number of Jordan curves $\gamma_{j}$. The winding number of $\gamma_{j}$ about any $z$ not on $\gamma_{j}$ is denoted by $n\left(\gamma_{j}, z\right)$, and if $z \notin \partial D$ we write

$$
n(\partial D, z)=\sum_{j} n\left(\gamma_{j}, z\right)
$$

Obviously,

$$
D=\{z: z \notin \partial D, n(\partial D, z) \neq 0\} .
$$

Finally, let $A$ and $B$ be disjoint, non-empty, compact subsets of $\mathbf{C}$. We put a rectangular grid on $\mathbf{C}$ which is fine enough so that no square in the grid meets both $A$ and $B$, and we let $\left\{Q_{j}\right\}$ be the set of those (closed) squares that meet $A$. Now let $\Omega$ be the interior of $\cup Q_{j}$ : then $\Omega$ is a bounded open set with a finite number of components $\Omega_{j}$, each being bounded by a finite number of Jordan curves, and (1) holds with $D$ replaced by $\Omega_{j}$. Further,

$$
A \subset \Omega, \quad B \cap \Omega=\emptyset, \quad \partial \Omega \cap(A \cup B)=\emptyset .
$$

We now give the

Proof of the Proposition. Let $D=\mathbf{C}_{\infty}-K$, and let $D_{j}$ be the components of $D$; Lemma 1 shows that each $D_{j}$ is simply connected. Next, it is easy to see that each component of $R^{-1}\left(D_{j}\right)$ is mapped by $R$ onto $D_{j}$, and because each $D_{j}$ is simply connected, we see that any component of $R^{-1}\left(D_{j}\right)$ is either a simply connected domain, or it is a domain of finite connectivity which contains a critical point of $R$ (for if such a component, say $\Delta$, does not contain a critical point then, by the monodromy theorem, the map $R$ of $\Delta$ onto $D_{j}$ is a homeomorphism). As $R^{-1}(D)$ is the union of the $R^{-1}\left(D_{j}\right)$, it follows that $R^{-1}(D)$ is the union of a finite number of multiply (but finitely) connected domains, say $M_{1}, \ldots, M_{t}$, and a number (possibly infinite) of simply connected domains $S_{j}$.

When there are no multiply connected domains $M_{j}$ present, all of the components of $R^{-1}(D)$ are simply connected and then Lemma 1 implies that the complement of $R^{-1}(D)$, namely $R^{-1}(K)$, is connected: thus the conclusion of the Proposition holds in this case.

We now assume that at least one domain $M_{j}$ exists, and we consider the minimal, and necessarily finite, set of components $E_{1}, \ldots, E_{q}$ of $R^{-1}(K)$ such that

$$
\bigcup \partial M_{j} \subset E_{1} \cup \cdots \cup E_{q}
$$


Next, we show that $E_{1}, \ldots, E_{q}$ are all of the components of $R^{-1}(K)$. Suppose, then, that $Q$ is another component of $R^{-1}(K)$ and write $E=E_{1} \cup \cdots \cup E_{q}$ : then $E$ and $Q$ are disjoint compact subsets of $R^{-1}(K)$, so from [5] (Theorem 5.6, p. 82), there are compact subsets $A$ and $B$ of $R^{-1}(K)$ such that

$$
A \cup B=R^{-1}(K), \quad A \cap B=\emptyset, \quad Q \subset A, \quad E \subset B .
$$

We may assume that $\infty \in R^{-1}(D)$; then $A$ and $B$ are disjoint, compact subsets of $\mathbf{C}$, so we can find an open set $\Omega$ (as described above) satisfying (2), and hence from (4), also

$$
\partial \Omega \subset R^{-1}(D)
$$

Now let $\Omega_{Q}$ be the component of $\Omega$ that contains the connected set $Q$. Using (3) and (4), we find that for each $r$,

$$
\partial M_{r} \subset E \subset B,
$$

and so we see from (2) that $\Omega_{Q}$ and $\partial M_{r}$ are disjoint. Now $\Omega_{Q}$ is arcwise connected, and this means that either $\Omega_{Q} \subset M_{r}$ or $\Omega_{Q} \cap M_{r}=\emptyset$. Now the first possibility cannot occur because if it does, then

$$
Q \subset \Omega_{Q} \subset M_{r} \subset R^{-1}(D)
$$

which violates the fact that $Q \subset R^{-1}(K)$; thus $\Omega_{Q}$ is disjoint from each $M_{r}$. As each $M_{r}$ is open, we deduce that the closure of $\Omega_{Q}$ is disjoint from $\cup M_{r}$.

As a consequence of this, each boundary component $\gamma_{j}$ (a Jordan curve) of $\Omega_{Q}$ lies in some simply connected domain $S_{m}$ for, by (5), it lies in $R^{-1}(D)$; thus one side of $\gamma_{j}$ lies in $S_{m}$, while the other side contains $R^{-1}(K)$ and each $M_{r}$. It follows that for any $z_{1}$ in $M_{r}$, and any $z_{2}$ in $Q$,

$$
n\left(\gamma_{j}, z_{1}\right)=n\left(\gamma_{j}, z_{2}\right)
$$

and hence that

$$
n\left(\partial \Omega_{Q}, z_{1}\right)=n\left(\partial \Omega_{Q}, z_{2}\right) \neq 0 .
$$

This shows that $z_{1}$ is in $\Omega_{Q}$, contrary to the fact that $\Omega_{Q}$ and $M_{r}$ are disjoint. It follows that no such component $Q$ exists, and so we have proved that

$$
R^{-1}(K)=E_{1} \cup \cdots \cup E_{q} .
$$

As $R^{-1}(K)$ is compact, so is each $E_{j}$, and hence $R\left(E_{j}\right)$ also: thus $R\left(E_{j}\right)$ is a closed subset of $K$. We shall now show that each $R\left(E_{j}\right)$ is relatively open 
in $K$ : then, as $K$ is connected, we find that $R\left(E_{j}\right)=K$. Clearly, this implies that $q \leq d$ and the proof of the Proposition will then be complete.

To show that $R\left(E_{j}\right)$ is relatively open in $K$, we take any $\zeta$ in $R\left(E_{j}\right)$, say $\zeta=R(w)$, where $w \in E_{j}$. We find a neighbourhood $N$ of $w$ not meeting any other $E_{i}$ (this is possible because $R^{-1}(K)$ has only finitely many components) and observe that

$$
K \cap R(N)=R\left(E_{j} \cap N\right) \subset R\left(E_{j}\right) .
$$

This shows that $R\left(E_{j}\right)$ is relatively open in $K$, and the proof of the Proposition is complete.

We end with the

Proof of the Theorem. Let $K$ be the set of points in $J$ at which infinitely many components of $J$ accumulate. Our first objective is to show that $J=K$ and to do this, we prove

(a) $K$ is closed;

(b) $K$ is completely invariant, and

(c) $K$ has at least three points.

With these, the minimality of $J$ shows that $J \subset K$, and hence that $K=J$.

Obviously, $K$ is closed, so (a) holds. By assumption, $J$ has infinitely many components so $K$ is not empty, and with this, (b) implies (c) (for, from the general theory of iteration, any non-empty finite completely invariant set lies in $F$ ). We shall now show that (b) holds.

First, take $\zeta$ in $K$, so there is a sequence $J_{1}, J_{2}, \ldots$ of distinct components of $J$ which accumulate at $\zeta$. Obviously, the components $R\left(J_{n}\right)$ accumulate at $R(\zeta)$, and from the Proposition we see that at most $d$ of the $J_{n}$ can map to any given component of $J$. We deduce that infinitely many components of $J$ accumulate at $R(\zeta)$, and hence that $R(K) \subset K$.

Next, take any $\zeta$ in $K$ and $w$ such that $R(w)=\zeta$ : then find neighbourhoods $U$ of $w$ and $V$ of $\zeta$ such that for an appropriate $k, R$ is a $k$-fold map of $U$ onto $V$. Again, there is a sequence $J_{1}, J_{2}, \ldots$ of distinct components of $J$ which accumulate at $\zeta$, and we may assume that all of these meet $V$. It follows that some component of each $R^{-1}\left(J_{n}\right)$ meets $U$, and these components must be distinct as the $J_{n}$ are. As $U$ and $V$ can be chosen arbitrarily small, this shows that $R^{-1}(K) \subset K$, and hence that (b) holds. We have now shown that $J=K$ and so, in particular, no component of $J$ is isolated.

It only remains to prove that $J$ has uncountably many components. We argue by contradiction, so suppose that the components of $J$ are $J_{1}, J_{2}, \ldots$ Now $J$ is a compact metric space, and $J$ is the countable union of the $J_{n}$ so, by Baire's category theorem not every $J_{n}$ is nowhere dense in $J$. We may suppose that $J_{1}$ is not, so the closure of $J_{1}$ has a non-empty interior (all in the relative topology on $J$ ). But $J_{1}$ is a component of $J$, so it is closed in $J$. We deduce that $J_{1}$ 
has a non-empty interior in $J$, and as this violates the statement at the end of the previous paragraph, we can conclude that $J$ must have uncountably many components.

\section{References}

[1] Blanchard, P.: Complex analytic dynamics on the Riemann sphere. - Bull. Amer. Math. Soc. 11, 1984, 85-141.

[2] Brolin, H.: Invariant sets under iteration of rational functions. - Ark. Mat. 6, 1965, 103-144.

[3] LyUBich, M.Y.: The dynamics of rational transforms: the topological picture. - Russian Math. Surveys 41, 1986, 43-117.

[4] MCMullen, C.: Automorphisms of rational maps. - Preprint, 1986.

[5] Newman, M.H.A.: Elements of the topology of plane sets of points. - Cambridge University Press, Cambridge, 1964.

University of Cambridge

Department of Pure Mathematics and Mathematical Statistics

16 Mill Lane

Cambridge CB2 1SB

England

Received 13 July 1990 